\title{
L'apprentissage organisationnel et son impact sur la performance des processus
}

À la suite des travaux de Mukerjee et al. (1998) identifiant les construits qui caractérisent I'apprentissage organisationnel dans le cadre du TQM et, montrant l'impact de l'apprentissage sur la performance organisationnelle, cet article étudie le cas des entreprises françaises certifiées ISO sur la base d'une étude empirique portant sur 132 processus industriels.

À quelques nuances, les résultats de l'étude corroborent ceux obtenus dans le cadre du TQM en ce qui concerne les construits de l'apprentissage malgré des différences de fond significatives des deux approches.

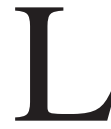

es programmes de Total Quality Management (TQM) massivement lancés et largement diffusés aux États-Unis depuis presque deux décennies donnent une place essentielle à la motivation du personnel, à la libération des énergies créatives, ainsi qu'aux démarches de progrès. Peu d'études font vraiment le lien entre ces approches et leurs conséquences sur le système d'acquisition et de capitalisation des connaissances. Pourtant les démarches qualité qui se sont développées un peu partout dans le monde sont devenues l'outil le plus pratiqué en matière de normes organisationnelles nécessitant une codification minimale des connaissances et pratiques à travers l'écriture de procédures associées aux principaux processus de l'entreprise. À la suite des travaux de Cole (1999) qui décri-

1. Nous remercions les entreprises qui font partie de la Fondation IECS de s'être prêtées au jeu du questionnaire et d'avoir accordé du temps à notre étude. 
vent de façon qualitative le lien entre des expériences TQM dans des grandes entreprises américaines et l'apprentissage, la recherche menée par Mukherjee et al. (1998) apporte un premier éclairage plus quantitatif en distinguant trois formes d'apprentissage. Sur la base d'une étude approfondie, portant sur une soixantaine de projets industriels, ces derniers montrent que les systèmes de pilotage qualité affectent les construits de l'apprentissage et cela à trois niveaux reprenant en partie la typologie de Kim (1993): l'apprentissage opérationnel, l'apprentissage conceptuel et un apprentissage de diffusion à travers l'organisation.

L'intérêt d'approcher l'apprentissage par le biais des démarches qualité provient du fait qu'aujourd'hui encore, peu d'entreprises mettent en pratique des systèmes explicites de management des connaissances alors qu'elles codifient abondamment celles-ci à travers les labels de certification. Les normes ISO 9000, par exemple, constituent un outil de management des connaissances qui conduit à une explicitation des savoirs sur l'organisation et à leur formalisation (Cowan et Foray, 2000; Cohendet et Steinmuller, 2000). Dans la nouvelle version 2000 des normes ISO, les savoirs dont il s'agit ne se limitent pas au caractère opérationnel de la maîtrise des processus mais touchent aussi au réservoir et à l'accès de connaissances vitales pour le développement stratégique de l'entreprise.

Utilisant une méthodologie similaire aux études américaines relatives aux démarches TQM, nous avons porté notre attention sur 132 processus de réalisation, de management et supports tous issus de démarches de certification ISO 9000 dans 24 entreprises situées dans la région Alsace. Les résultats auxquels nous aboutissons confirment à quelques nuances près les travaux américains. La famille des normes ISO s'étant dans l'esprit rapprochée des prix de qualité totale en autorisant des évolutions du système conformes à l'amélioration continue, leur mise en œuvre affecte tout autant le système de management des connaissances que les démarches TQM. Elles trouvent peut-être en tant que base documentaire des principaux processus de l'entreprise une fonction inattendue dans le champ du management, celle d'une gestion explicite des éléments de connaissance nécessaires au développement stratégique de l'entreprise.

\section{I. - PROBLÉMATIQUE DE LA RECHERCHE}

Les recherches sur lesquelles nous basons notre étude (Mukherjee et al., 1998) s'inscrivent dans une filiation de travaux sur l'apprentissage largement inspirés des démarches de qualité totale. En effet, nous retrouvons à l'origine la fameuse séquence Plan-Do-Check-Act de Shewhart (1989) et Deming (1991) permettant d'articuler les deux dimensions de l'apprentissage au cœur de notre raisonnement: les apprentissages conceptuel et opérationnel. Cette séquence se retrouve dans la définition de l'apprentissage individuel donnée par Kim (1993). Selon cet auteur, l'individu dispose dans ses modèles mentaux de routines qui alimentent l'apprentissage opérationnel. Par ailleurs, l'individu s'appuie sur des modes de représentation ou « frameworks » qu'il mobilise afin de réaliser un apprentissage sur le plan conceptuel. Ce sont ces deux formes d'apprentissage qui constituent la base des changements au niveau de 
l'organisation à travers l'expérimentation dans les entreprises. Mukherjee et Van Wassenhove (1997) montrent que deux formes de connaissances sont à l'œuvre dans l'entreprise, l'une basée sur l'expérience, l'autre sur des schémas acquis plus formellement à travers le système éducatif et la formation continue par exemple. Les auteurs établissent un lien avec les démarches qualité qui codifient et garantissent la pérennité en quelque sorte des connaissances accumulées dans l'entreprise.

Dans des travaux basés sur l'étude clinique de deux PME nous avons montré (Bénézech et al., 2001) par ailleurs que tout se passait comme si l'apprentissage du code qualité permettant de rendre visibles les savoir-faire opérationnels (le know how) dans les processus de l'entreprise ouvrait une seconde perspective d'apprentissage plus profonde affectant les couches de la connaissance conceptuelle (le know why). Toutefois le passage de l'un à l'autre des modes d'acquisition de connaissances n'est pas acquis d'avance, et il nous semble que c'est précisément ce passage qui permettrait de faire un vrai partage entre des démarches qualité superficielles et bureaucratiques avec des démarches qualité débouchant sur une vraie dynamique d'apprentissage pour l'entreprise. Le lien entre ces deux formes d'apprentissage a fait l'objet d'une attention particulière dans les travaux relatés ci-après.

Sur la base d'un questionnaire utilisant une échelle de Lickert à cinq niveaux et administré sur soixante deux projets industriels, Mukherjee et al. (1998) montrent comment les démarches de TQM proposent un cadre de connaissance débouchant sur une codification des savoirs. Cette étude quantitative offre une perspective intéressante sur les liens encore flous souvent évoqués entre, d'une part, les démarches de qualité totale très développées aux États-Unis et, d'autre part, les formes d'apprentissage conceptuel et opérationnel ainsi que sur la capacité de l'entreprise à transférer ces apprentissages au cœur de l'organisation (facteur scope). En fait l'analyse des coefficients de corrélation permet d'identifier trois dimensions ou « construits » qui caractérisent le processus d'apprentissage organisationnel comme le montre le tableau 1 ci-après. Les auteurs considèrent que les trois premières variables de ce tableau forment un construit de l'apprentissage lié au facteur scope. Ils montrent en effet que la mise en œuvre des projets TQM sur plusieurs fonctions et départements permet de mieux maîtriser les systèmes complexes en facilitant notamment une compréhension des relations de cause à effet mais aussi permet la diffusion et l'élargissement du champ de l'apprentissage conceptuel et opérationnel généré localement. Ce facteur de diffusion et de champ d'application des apprentissages est appelé scope par les auteurs.

De même, l'utilisation de modèles scientifiques, d'outils statistiques, de diagrammes d'Ishikawa dans le cadre des démarches TQM caractérise l'apprentissage conceptuel (les trois variables suivantes du tableau). L'apprentissage opérationnel se caractérise par le suivi des expérimentations et la modification des variables d'action (les deux dernières variables). 


\begin{tabular}{|l|c|c|c|c|}
\hline \multicolumn{5}{|c|}{ Tableau 1 } \\
\multicolumn{2}{|c|}{ ANALYSE FACTORIELLE SUR 62 PROJETS } \\
\hline & $\begin{array}{c}\text { Facteur } \\
\text { Scope }\end{array}$ & $\begin{array}{c}\text { Apprentissage } \\
\text { conceptuel }\end{array}$ & $\begin{array}{c}\text { Apprentissage } \\
\text { opérationnel }\end{array}$ & $\begin{array}{c}\text { Qualité de la } \\
\text { représentation }\end{array}$ \\
\hline Groupe interfonctionnel & $\mathbf{0 , 9 2}$ & 0,15 & 0,06 & 0,88 \\
\hline Mise en œuvre interdépartemental & $\mathbf{0 , 8 1}$ & 0,06 & 0,22 & 0,72 \\
\hline Champ d'application du projet & $\mathbf{0 , 7 1}$ & 0,38 & 0,02 & 0,65 \\
\hline Utilisation d'outils statistiques & 0,10 & $\mathbf{0 , 8 1}$ & 0,15 & 0,69 \\
\hline Utilisation de modèles scientifiques & 0,50 & $\mathbf{0 , 7 0}$ & 0,16 & 0,77 \\
\hline Niveaux du diagramme d'Ishikawa & 0,51 & $\mathbf{0 , 6 5}$ & 0,15 & 0,71 \\
\hline Suivi des expérimentations & 0,25 & 0,12 & $\mathbf{0 , 9 1}$ & 0,91 \\
\hline Modification des variables d'action & $-0,07$ & 0,60 & $\mathbf{0 , 6 6}$ & 0,79 \\
\hline Variance expliquée & 2,61 & 2,12 & 1,38 & 6,12 \\
\hline Variance expliquée en pourcentage & 32,7 & 26,3 & 17,3 & 76,3 \\
\hline
\end{tabular}

Source: Mukherjee et al. (1998).

Ces auteurs montrent que ces trois construits ont un impact sur la performance des projets. Par exemple, l'impact des construits de l'apprentissage sur le changement des règles d'attention qui permet de faire évoluer les comportements individuels, et, partant, de l'organisation dans son ensemble découle de cette recherche. Ce changement semble plus propice lorsque l'apprentissage conceptuel réalisé est suivi d'un apprentissage opérationnel. Ces travaux précisent le sens des relations sur des bases plus solides que les pures convictions managériales d'amélioration continue évoquées la plupart du temps par les entreprises. Deux perspectives de recherche nous sont alors apparues indispensables. Une telle étude empirique utilisant les mêmes outils et la même rigueur d'analyse est-elle possible dans le contexte des démarches qualité des entreprises françaises. D'autre part, les normes ISO étant plutôt la référence dans le contexte français, retrouve t-on le même type de résultat sur un échantillon français de différents processus industriels? En d'autres termes, les démarches qualité introduites par le référentiel européen ISO insufflent-elles la même dynamique d'apprentissage du fait des efforts fournis par les entreprises en matière de codification des connaissances, ce dernier facteur nous apparaissant comme essentiel dans les logiques de changement organisationnel (voir à ce sujet par exemple Lambert, Loos-Baroin, 2004).

En clair, la question à laquelle nous nous attelons et qui anime de nombreux groupes de réflexion dans les entreprises est la suivante: 
Dans les entreprises certifiées ISO 9000, pouvons-nous identifier les mêmes construits qui caractérisent l'apprentissage organisationnel et quel est l'impact de ces construits sur la performance des processus?

Cette question trouve sa justification dans le fait que même si les normes ISO 9000 se sont rapprochées de la qualité totale, des différences assez significatives existent entre les deux approches; ce qui peut laisser penser a priori que l'une ou l'autre offre des opportunités d'apprentissage différentes. Nous revenons sur les différences entre les deux approches de la qualité à la section suivante.

\section{II. - DIFFÉRENCES ENTRE ISO 9000 ET TQM}

Diffusées pour la première fois en 1987, la série des normes ISO a connu des évolutions avec les versions 94 et 2000. Bien que la version 2000 se soit beaucoup rapprochée du TQM, des différences assez significatives existent encore entre les deux.

La politique qualité dans le TQM est centrée sur la qualité des produits et services et sur la réalisation de profits alors que l'ISO vise plutôt à garantir la reproductibilité de la qualité décrite dans le référentiel qualité et la lisibilité d'un système qualité par un référentiel commun. La qualité selon l'ISO a en effet une orientation externe, nécessitant la reconnaissance par un organisme certificateur alors que le TQM est orienté efficience interne. Certaines entreprises adoptent en effet le TQM sans rechercher l'obtention d'un prix qualité totale mais le font dans le but d'avoir une évaluation objective de leur système. La mesure du progrès mise en œuvre chaque année devient la motivation principale d'une telle démarche. En conséquence, la documentation est facultative dans le TQM, alors qu'elle occupe une place importante dans la certification ISO, et ce dans le but de donner la preuve de la capacité de l'organisme à reproduire le même niveau de qualité. On assiste donc à une multitude de méthodes pour conduire le TQM tandis que pour l'ISO, on a un référentiel commun et public. Contrairement à l'ISO où la direction fixe les objectifs à atteindre puis recherche l'implication de tout le personnel, avec le TQM, ce sont les groupes de travail qui fixent leurs propres objectifs. Le management participatif est donc primordial dans le TQM tandis qu'il se limite à rechercher l'implication du personnel dans l'ISO. Il faut noter également que les audits internes et la traçabilité des produits sont obligatoires dans le référentiel ISO alors que ces notions sont presque inexistantes dans le TQM.

Les deux approches sont similaires sur beaucoup de points, entre autres, l'adoption d'une politique qualité, l'approche processus de l'organisation, l'amélioration continue, la maîtrise des procédés, les actions correctives et préventives, la mesure, la formation, etc.

\section{III. - MÉTHODOLOGIE ET HYPOTHĖSES DE TRAVAIL}

Pour répondre à cette problématique, nous avons adressé un questionnaire aux responsables qualité de 98 entreprises manufacturières alsaciennes de tailles variées, certifiées ISO 9000. Nous avons ainsi obtenu 24 réponses soit un taux de retour d'environ $25 \%$. Dans chaque entreprise, nous avons identifié en moyenne six processus; ce qui 
nous a permis d'obtenir 132 processus constituant nos individus statistiques. Le traitement des données a été fait à l'aide du logiciel SPSS.

La norme ISO 9001 version 2000 permet d'identifier principalement trois types de processus. Les processus de réalisation interviennent en amont dans la détection $\mathrm{du}$ besoin du client, puis dans la réalisation du produit et en aval dans la satisfaction de ces besoins. Les processus support fournissent les ressources (humaines, matérielles, financières, etc.) nécessaires aux autres processus et les processus de management ou de pilotage contribuent à déterminer la politique qualité, à fixer les objectifs et à les déployer dans toute l'organisation. A priori, la maîtrise et la réalisation d'une bonne performance des processus de réalisation comme la conception, la recherche-développement, la fabrication, exigent de grandes compétences scientifiques et une certaine capacité de modélisation et souvent de conception (connaissances conceptuelles). Il nous semble également que les processus support comme la maintenance, la sécurité, la logistique pourraient, à la limite, être mâ̂trisés grâce à un suivi régulier des instructions, devenant routines par la suite (connaissances opérationnelles). Quant aux processus de management, leur performance dépend d'une bonne expérience en leadership et d'une grande capacité de communication et de partage de l'information et de la connaissance (maîtrise du facteur scope).

L'apprentissage conceptuel pourrait donc avoir un impact très significatif sur la performance à long terme et à court terme des processus de réalisation. L'apprentissage opérationnel pourrait permettre de réaliser une bonne performance des processus support. La capacité à diffuser la connaissance créée localement et donc à élargir le champ de l'apprentissage (scope) pourrait jouer un rôle très important dans la performance des processus de management. Cependant, malgré les différences entre les deux approches, les caractéristiques latentes de l'apprentissage organisationnel pourraient être les mêmes, que le référentiel soit l'ISO ou le TQM; d'où les deux hypothèses suivantes:

Hypothèse 1. Les construits qui caractérisent l'apprentissage organisationnel sont les mêmes dans les référentiels ISO et TQM.

Hypothèse 2. L'impact de chaque construit sur la performance des processus dépend toutefois de la nature des processus.

Avant de procéder au test de ces deux hypothèses, quelques constats d'ordres généraux s'imposent.

\section{IV. - RÉSULTATS ET INTERPRÉTATION}

\section{Constats d'ordres généraux}

Revenant sur certains constats généraux suite à notre enquête, cette section permet de jeter un regard sur les pratiques réelles des entreprises et de voir leur éventuelle adéquation avec les positions défendues par la littérature.

Les objectifs poursuivis par les entreprises à travers les démarches qualité

Il apparaît clairement de notre étude que les deux objectifs prioritaires poursuivis par les entreprises à travers la mise en œuvre des démarches qualité sont la recherche de la satisfaction des clients et l'amélioration des résultats. 
En effet, 20 entreprises parmi les 24, soit plus de $83 \%$, ont répondu que l'un des deux objectifs prioritaires poursuivis à travers le SMQ était bien la satisfaction des clients. Ce résultat concorde parfaitement avec l'esprit des démarches qualité qui vise en effet à satisfaire les besoins explicites et latents des clients.

Plus de la moitié des entreprises citent également l'amélioration des résultats parmi leurs deux principaux objectifs. Les démarches qualité peuvent en effet permettre de réaliser l'objectif de rentabilité grâce à la maîtrise des coûts de la non-qualité, l'application de méthode comme l'analyse de la valeur, l'amélioration des positions concurrentielles et l'augmentation des marges grâce à la qualité des produits, etc. Par contre, le développement des savoirfaire et de l'innovation ne constituent pas véritablement un objectif prioritaire pour la plupart des entreprises qui se lancent dans les démarches de certification aux normes ISO 9000. En effet, seules trois entreprises sur vingt quatre disent poursuivre un tel objectif. Ce résultat montre clairement que l'attention n'est pas directement portée sur l'apprentissage organisationnel et la gestion des connaissances lors de l'élaboration et de la mise en œuvre des démarches qualité. Mukherjee et al. (1998) notent que la littérature qui traite du TQM ne s'est pas beaucoup intéressée à l'apprentissage organisationnel, bien que l'adoption de ce système de management soit une approche particulièrement apprenante. Nous pouvons ajouter que pour ce qui est des démarches qualité de type ISO, même les praticiens n'en font pas une préoccupation systématique. Certaines entreprises citent la clarification des responsabilités et la réorganisation parmi leurs objectifs prioritaires. Qu'ils soient ou non des objectifs poursuivis, la clarification des responsabilités des acteurs et le changement organisationnel se produisent généralement lors de la mise en œuvre du SMQ. La moitié des entreprises constate en effet que le SMQ a été une occasion pour mieux clarifier les responsabilités de chacun et pour réorganiser tout le système.

Il apparaît également que l'objectif d'accès à de nouveaux marchés n'est pas prioritaire pour les entreprises lorsqu'elles se lancent dans les démarches de certification: seules deux entreprises parmi les vingt quatre disent poursuivre un tel objectif.

Lorsque les démarches qualité sont imposées de l'extérieure, par un client important par exemple, la certification devient une fin en soi. Cela peut donner naissance à un système qualité de façade. Par contre, lorsque l'adoption de l'ISO répond à un besoin interne de réorganisation ou fait suite aux orientations stratégiques de l'entreprise, bien comprises par le personnel en matière de qualité, le SMQ peut contribuer à changer le regard que les responsables portent sur leur entreprise, occasionner des opportunités d'apprentissage en double boucle, et améliorer la perception que le personnel a de la qualité. Il faut noter en effet que la perception de la qualité n'est pas toujours positive. Le responsable qualité d'une entreprise d'électricité affirme à cet effet: « il y a un bruit de fond sur la lourdeur du système et la documentation excessive que nous devons combattre journellement ». En fait, ce «bruit de fond » semble exister dans beaucoup d'entreprises. Suite à une enquête « extra muros » auprès d'une cinquantaine de personnes travaillant dans des entreprises certifiées ISO 9000 version 94 à Laval au Québec, Boiral (2002) constate quatre types de perceptions de la qualité 
les « intégrateurs qualité » $(10 \%)$ qui sont en fait des responsables qualité, les « adeptes engagés » (20 à $25 \%)$, les « dissidents » $(20 \%)$ et les « extravertis rituels » (45 à 50 \%). Les extravertis rituels adhèrent seulement en surface au SMQ, le voyant surtout comme un label commercial, donc un mal nécessaire, tandis que les dissidents affirment de façon assez catégorique leur opposition. De même, Igalens (1998) constate que le développement des pratiques de gestion de la qualité est négativement associé à l'évaluation par les DRH, du niveau de mobilisation des ressources humaines; ce qui infirme le discours habituel qui soutient que la qualité satisfait le personnel avant de satisfaire le client. Le passage à la version 2000 devrait contribuer à réduire cette perception plutôt négative de la qualité.

Qu'en est-il du potentiel des démarches qualité à stimuler la création de nouveaux produits?

\section{Démarches qualité: opportunité}

d'améliorer la qualité des produits

existants ou de créer de

nouveaux produits?

Il ressort de notre étude que le SMQ est plus une opportunité d'améliorer la qualité des anciens produits qu'une occasion pour en créer de nouveaux. De façon générale, les entreprises reconnaissent que la qualité des produits s'est améliorée suite à la mise en œuvre des démarches qualité. En effet, grâce à son potentiel de réorganisation, d'implication du personnel et de rigueur et surtout grâce aux programmes d'amélioration continue des processus, le SMQ peut être un moyen efficace d'amélioration de la qualité des anciens produits. En revanche, le potentiel des démarches qualité à générer de nouvelles idées conduisant par exemple à la conception de nouveaux produits et à des dépôts de brevets est assez limité. Environ $70 \%$ des entreprises interrogées ne reconnaissent pas un tel potentiel dans les démarches qualité. Ce résultat indique que les programmes d'amélioration continue autorisent plus un apprentissage de type incrémental qu'exploratoire. C'est l'apprentissage de type « exploratoire » qui permet en effet de générer des idées tout à fait nouvelles, pouvant déboucher sur la création de nouveaux produits.

\section{Autonomie du personnel et contribution des cadres moyens à la mise en cuvre du $S M Q$}

Les cadres moyens ont été l'objet de certaines critiques assez virulentes, par exemple Peters (1987) et Drucker (1988). A contrario, Nonaka et Takeuchi (1997) leur reconnaissent un grand rôle dans le processus de création et de diffusion de connaissances. Pour ce qui est des démarches de certification aux normes ISO 9000, les études de cas de Bénézech $e t$ al. (2001), Lambert et Loos-Baroin (2004), ont montré que le personnel de niveau intermédiaire joue un rôle très important dans le processus d'élaboration et de mise en œuvre du système documentaire qualité.

Nos résultats montrent que la contribution des cadres moyens à la conception et à la mise en œuvre du SMQ est assez moyenne. En effet, $50 \%$ des entreprises de notre échantillon constatent une contribution plutôt ou assez majeure de leurs cadres moyens, contre $37 \%$ qui notent que les cadres moyens n'ont eu que peu de contribution; les autres entreprises ne s'étant pas prononcées. 
Pour ce qui est de l'autonomie du personnel, nos résultats permettent de dire que celui-ci est assez autonome dans la conception et la mise en œuvre des solutions d'amélioration de processus. Comme l'avançait en son temps Dubreuil (1959), l'autonomie permet d'éviter les frustrations et les problèmes psychologiques liés à la subordination au chef d'entreprise. Selon cet auteur, l'autonomie permet également au personnel de libérer son génie créatif lors de la recherche et de la mise en œuvre des solutions. En faisant naître un sentiment de responsabilité et de satisfaction, l'autonomie accordée au personnel peut se traduire par une plus grande implication et une amélioration de la qualité des produits.

Dans la section suivante, nous allons identifier les construits qui caractérisent l'apprentissage organisationnel.

\section{Les construits de l'apprentissage organisationnel}

Avant d'identifier les dimensions théoriques de l'apprentissage organisationnel, revenons sur ce thème pour présenter ces deux courants et le distinguer de la gestion des connaissances qui lui est très proche.

La littérature sur l'apprentissage organisationnel peut être divisée en deux courants selon Argyris et Schön (2003) : le courant de l'organisation apprenante et celui de l'apprentissage organisationnel. Le courant de l'organisation apprenante est « prescriptive, orientée vers la pratique et essentiellement produite par les consultants et les gens de terrain » tandis que la littérature sur l'apprentissage organisationnel « est essentiellement sceptique et savante, fruit de recherches universitaires » (p. 236).

Les auteurs soutiennent que dans la littérature sur l'organisation apprenante, on n'y retrouve les notions d'adaptabilité organisationnelle, de flexibilité, d'évitement des pièges de la stabilité, de capacité humaine à apprendre au service d'objectifs organisationnels, tandis que la littérature sur l'apprentissage organisationnel, sceptique et volontairement éloigné de la pratique, se concentre sur la définition même de l'apprentissage organisationnel, de la possibilité qu'une organisation puisse apprendre, des types d'apprentissage organisationnel possibles, de l'intérêt et de la pertinence même du thème. Mais tous les deux courants s'intéressent à la capacité des organisations à tirer des inférences valides et utiles de leurs expériences et de leurs observations et à convertir celles-ci en actions qui portent leurs fruits, concluent-ils.

Si l'apprentissage, individuel ou organisationnel est « le processus qui consiste à améliorer des actions par un approfondissement des connaissances et de la compréhension » comme le soutiennent Fiol et Lyles (1985, p. 803), la gestion des connaissances est le « processus de création, d'enrichissement, de capitalisation et de diffusion des savoirs qui implique tous les acteurs de l'organisation, en tant que consommateurs et producteurs » (Prax, 2000, p. 17). On comprend de ces deux définitions que l'apprentissage organisationnel s'intéresse en premier lieu à l'acquisition de la connaissance et à l'adaptation organisationnelle suite aux expériences et aux connaissances acquises, tandis que la gestion des connaissances s'intéresse certes au processus d'acquisition mais également à l'enrichissement, la capitalisation et la diffusion des connaissances. Notons toutefois que la question du passage entre apprentissage individuel et organisationnel, donc finalement de la diffusion de l'apprentissage, fait l'objet d'une grande attention dans la littérature savante sur 
l'apprentissage organisationnel, par exemple Kim (1993). C'est dire que les frontières entre apprentissage organisationnel et gestion des connaissances ne sont pas faciles à délimiter. C'est pour cette raison que Cole (1999) les considère comme des cousins.

Pour tester la validité de la première hypothèse qui stipule que les construits de l'apprentissage sont les mêmes dans les référentiels ISO et TQM, nous identifierons ci-dessous les construits de l'apprentissage organisationnel dans les entreprises certifiées ISO. Pour ce faire, nous utiliserons la méthode de l'analyse en composantes principales avec rotation varimax.

\section{Analyse en composantes principales sur 132 processus}

Les variables entrant dans l'analyse en composantes principales sont les neuf pre- mières variables du tableau 2 ci-dessous. Les trois dernières sont utilisées dans la régression multiple en tant que variables dépendantes. Toutes ces variables ont été mesurées sur une échelle de Likert à cinq points. Dans cette première analyse, nous étudierons d'abord la corrélation des variables entre elles, puis la corrélation des variables avec les axes factoriels.

a) Analyse de la corrélation entre les variables de l'étude

Comme le montre le tableau 2, des corrélations relativement fortes existent entre certaines variables de notre étude. On remarquera d'ores et déjà que, de façon générale, les variables sont positivement corrélées entre elles.

D'une part, nous avons une corrélation forte $(0,65)$ entre les variables «processus

\begin{tabular}{|c|c|c|c|c|c|c|c|c|c|c|c|c|}
\hline \multicolumn{13}{|c|}{ Tableau 2} \\
\hline $\begin{array}{l}\text { Corrélations entre } \\
\text { variables }\end{array}$ & $\begin{array}{c}\text { Proces. } \\
\text { inter- } \\
\text { fonc- } \\
\text { tionnel }\end{array}$ & $\begin{array}{c}\text { Syn. } \\
\text { Proces- } \\
\text { sus }\end{array}$ & $\begin{array}{c}\text { Outils } \\
\text { stat. }\end{array}$ & $\begin{array}{c}\text { modèles } \\
\text { scien- } \\
\text { tifiq. }\end{array}$ & $\begin{array}{c}\text { Dia- } \\
\text { gramme } \\
\text { Ishikaw. }\end{array}$ & $\begin{array}{c}\text { Variable } \\
\text { d'ac- } \\
\text { tion }\end{array}$ & $\begin{array}{c}\text { Mise } \\
\text { à jour } \\
\text { procé- } \\
\text { dures }\end{array}$ & $\begin{array}{c}\text { Mise } \\
\text { à jour } \\
\text { instruc } \\
\text { tions }\end{array}$ & $\begin{array}{c}\text { Amél. } \\
\text { proce- } \\
\text { ssus }\end{array}$ & $\begin{array}{c}\text { Atteinte } \\
\text { objec- } \\
\text { tifs } \\
\text { CT }\end{array}$ & \begin{tabular}{|c|} 
Atteinte \\
objec- \\
tifs \\
LT
\end{tabular} & $\begin{array}{l}\text { Capac. } \\
\text { spéci- } \\
\text { fier } \\
\text { l'impact }\end{array}$ \\
\hline Processus interfonctionnel & 1,00 & ,650 & ,036 &,- 012 & ,056 & , 109 & ,216 &, 155 & , 105 &, 155 &, 115 &,- 094 \\
\hline Synergie entre processus & & 1,00 & ,296 &, 350 &, 317 &, 319 & 419 & ,455 & ,096 & ,255 & ,522 & ,274 \\
\hline Outils statistiques & & & 1,00 & ,606 & 560 & ,464 &, 353 & ,318 &, 129 & ,222 & ,382 & ,439 \\
\hline $\begin{array}{l}\text { Utilisation de modèles } \\
\text { scientifiques }\end{array}$ & & & & 1,00 & ,468 & ,404 &, 161 &, 221 &, 032 &,- 014 &, 268 & ,388 \\
\hline $\begin{array}{l}\text { Nbre niveaux diagramme } \\
\text { Ishikawa et autres modèles }\end{array}$ & & & & & 1,00 &, 507 &, 233 & ,472 & 297 &, 224 & ,293 & 384 \\
\hline $\begin{array}{l}\text { Modification des variables } \\
\text { d'action }\end{array}$ & & & & & & 1,00 &, 238 &, 366 &, 372 & ,398 & ,492 & ,478 \\
\hline Mise à jour procédures & & & & & & & 1,00 &, 484 &, 373 & ,494 & ,613 & , 173 \\
\hline $\begin{array}{l}\text { Mise à jour instructions } \\
\text { de travail }\end{array}$ & & & & & & & & 1,00 &, 359 &, 252 & ,475 &, 334 \\
\hline Amélioration processus & & & & & & & & & 1,00 &, 359 &, 308 &,- 126 \\
\hline $\begin{array}{l}\text { Atteinte des objectifs } \\
\text { à court terme }\end{array}$ & & & & & & & & & & 1,00 & ,737 & ,386 \\
\hline $\begin{array}{l}\text { Atteinte des objectifs } \\
\text { à long terme }\end{array}$ & & & & & & & & & & & 1,00 &, $\mathbf{5 7 0}$ \\
\hline $\begin{array}{l}\text { Capacité à spécifier } \\
\text { l'impact de variables }\end{array}$ & & & & & & & & & & & & 1,00 \\
\hline
\end{tabular}


interfonctionnel » et «synergie entre les processus ». Plus un processus fait intervenir beaucoup de fonctions et départements, plus des synergies sont générées à travers les interactions. D'autre part, nous observons une corrélation de 0,60 entre les variables « utilisation d'outils statistiques » et « utilisation de modèles scientifiques ». De même, une corrélation de 0,56 est observée entre « utilisation d'outils statistiques » et " nombre de niveaux du diagramme d'Ishikawa ». Mukherjee et al. (1998) notent que ces outils contribuent à identifier et à mesurer les causes sous-jacentes aux problèmes et à conceptualiser des solutions. Ces outils constituent donc une dimension de l'apprentissage conceptuel (Kim, 1993). Par ailleurs, nous remarquons une corrélation de 0,57 entre l'atteinte des objectifs à long terme et la capacité à spécifier l'impact des données d'entrée sur les résultats de processus. Dans des systèmes de production complexes, la compréhension des relations de causes à effet est non seulement un préalable mais aussi le signe d'un apprentissage conceptuel.

La corrélation de 0,74 entre l'atteinte des objectifs à long terme et à court terme est assez intuitive. On s'attend en effet à ce qu'une organisation qui atteint ses objectifs à court terme, réalise au final ses objectifs stratégiques. Toutefois, cette relation de cause à effet n'est pas systématique. Un accent particulier mis sur les objectifs à court terme pourrait même faire perdre de vue les objectifs stratégiques de l'organisation.

b) Analyse de la corrélation des variables aux axes factoriels

Les corrélations des variables aux axes sont résumées dans le tableau 3 ci-après.

\section{Premier axe : apprentissage conceptuel}

Comme le montre le tableau III ci-dessous, trois variables sont fortement corrélées au premier axe factoriel. Il s'agit de l'utilisation d'outils statistiques (corrélation de $0,84)$, de l'utilisation de modèles scientifiques $(0,86)$ et du nombre de niveaux du diagramme d'Ishikawa $(0,67)$. Le diagramme d'Ishikawa (diagramme de causes à effets) détaillé, comme son nom l'indique, permet de déterminer les causes et souscauses d'un problème. L'utilisation d'outils statistiques (diagramme de Pareto, histogramme, techniques d'échantillonnage) permet de déterminer l'ampleur de ces causes et effets. L'utilisation de modèles scientifiques permet enfin de concevoir des solutions sous forme de modèles théoriques. Ces trois variables constituent donc une dimension du processus d'apprentissage conceptuel (Kim, 1993). Notre premier axe peut donc s'interpréter comme un axe d'apprentissage conceptuel.

Certaines remarques s'imposent toutefois en ce qui concerne l'ampleur de l'apprentissage conceptuel dans les organisations certifiées ISO. Plus de 50\% des entreprises n'utilisent aucun modèle scientifique pour la recherche de solution d'amélioration des processus. Lors des démarches qualité, l'utilisation de modèles scientifiques comme les plans d'expérience, est très marginale. Quant aux outils statistiques, leur utilisation est certes plus importante que celle des modèles scientifiques du fait de leur préconisation dans le cadre des démarches qualité, mais elle reste néanmoins modérée. De même, pour ce qui est du diagramme d'Ishikawa, son utilisation est très limitée parmi les entreprises certifiées ISO 9000. Plus de $66 \%$ des entreprises interrogées affirment ne pas utiliser 


\begin{tabular}{|c|c|c|c|c|}
\hline \multicolumn{5}{|c|}{$\begin{array}{c}\text { Tableau } 3 \\
\text { COORDONNÉES DES VARIABLES APRÈS ROTATION SUR LES TROIS PREMIERS } \\
\text { AXES ET QUALITÉ DE LA REPRÉSENTATION (132 PROCESSUS) }\end{array}$} \\
\hline \multirow[b]{2}{*}{ Variables } & \multicolumn{3}{|c|}{ Composantes } & \multirow[b]{2}{*}{$\begin{array}{l}\text { Qualité de la } \\
\text { représentation }\end{array}$} \\
\hline & $\begin{array}{l}\text { Axe 1: } \\
\text { Apprentissage } \\
\text { Scope }\end{array}$ & $\begin{array}{l}\text { Axe 2: } \\
\text { Facteur } \\
\text { Scope }\end{array}$ & $\begin{array}{l}\text { Axe } 3: \\
\text { Apprentissage } \\
\text { opérationnel }\end{array}$ & \\
\hline Processus interfonctionnel &,- 05 &, 84 & 02 & ,70 \\
\hline Synergie entre processus &, 33 &, 85 &, 11 &, 85 \\
\hline Utilisation d'outils statistiques &, 84 & ,09 &, 15 &, 74 \\
\hline Utilisation de modèles scientifiques & ,86 &, 16 &,- 07 &, 77 \\
\hline $\begin{array}{l}\text { Nombre de niveaux du diagramme } \\
\text { Ishikawa ou degré de détail } \\
\text { des autres modèles }\end{array}$ & ,67 & ,20 &, 31 &, 59 \\
\hline Modification des variables d'action & ,48 &,- 13 & ,46 & ,46 \\
\hline Mise à jour des procédures &, 21 &, 48 &, 55 &, 58 \\
\hline Mise à jour des instructions de travail &, 35 &, 37 &, 60 & ,62 \\
\hline Amélioration continue des processus &,- 03 &,- 01 & ,88 &, 77 \\
\hline Variance expliquée & 3,54 & 1,35 & 1,19 & 6.08 \\
\hline Variance expliquée en $\%$ & 39,33 & 15,00 & 13,22 & 67,55 \\
\hline
\end{tabular}

cet outil. Les entreprises qui l'utilisent s'arrêtent en général à deux souscauses. Notons qu'un tel résultat avait déjà été observé dans les entreprises américaines dans les années 1990 (Spencer, 1994).

Les outils statistiques et modèles scientifiques étant peu utilisés lors de la mise en œuvre du SMQ, nous pouvons conclure que la certification aux normes ISO n'affecte pas en priorité les connaissances conceptuelles. Mukherjee et al. (1998) constatent également une utilisation marginale de ces outils et modèles dans le cadre des programmes TQM.

\section{Deuxième axe: facteur scope}

Comme on peut le constater dans le tableau 3, les variables « processus interfonctionnel » et « synergie entre processus » sont fortement corrélées avec le deuxième axe factoriel (respectivement 0,84 et 0,85 ). Lorsque l'amélioration d'un processus implique plusieurs fonctions et départements et que, de plus, des interactions importantes et des synergies existent entre ce processus et les autres, l'apprentissage généré peut s'étendre très facilement à toute l'organisation. Ce deuxième axe peut donc s'interpréter comme un facteur scope (Mukherjee et al., 1998), permettant de 
déployer le champ de l'apprentissage à toute l'organisation. Le facteur scope dans l'étude de Mukherjee et al. (1998) a été mesuré à travers les groupes interfonctionnels, la mise en œuvre de solutions de façon transversale et le champ d'application des projets. Ces auteurs soutiennent que dans les organisations aux systèmes de production complexes, la mise en œuvre locale d'un projet n'offre pas d'opportunités de comprendre ses résultats sur le système dans son ensemble. Dans ce cas, plus le champ d'application du projet est large (scope), plus il est possible de lier causes et effets. L'apprentissage conceptuel et le facteur scope permettent donc de mieux comprendre les relations de causes à effets et de mieux maîtriser le système complexe dans son ensemble. C'est cette capacité à appréhender le système (complexe) qui est mesuré en termes de « capacité à spécifier l'impact des inputs sur les outputs », c'est-à-dire la compréhension des lois qui gouvernent le système dans son ensemble. Ainsi nous avons pu constater dans une entreprise fabriquant des appareils électroniques (ondulateurs et appareillages) la mise en place de steering committee. Les responsables conception, marketing et production des deux activités se réunissent régulièrement non seulement dans le cadre de projets communs mais aussi pour échanger et transférer des expériences, des expertises et des compétences. De telles pratiques permettent de diffuser les connaissances locales à toute l'organisation et d'étendre le champ d'application des projets et des solutions.

Le caractère interfonctionnel et synergique de certains processus ainsi que les groupes de projets interfonctionnels rassurent donc sur le fait que l'apprentissage conceptuel et ou opérationnel généré lors des démarches de certification et des programmes d'amélioration de processus ne restera pas local mais s'étendra à toute l'organisation sous forme de modèles mentaux (Senge, 1990) partagés et de routines organisationnelles.

\section{Troisième axe : apprentissage opérationnel}

Les variables « mise à jour des procédures », « mise à jour des instructions de travail », « amélioration continue des processus » sont les plus corrélées au troisième axe factoriel (cf. tableau 3). Des mises à jour fréquentes des procédures et des instructions de travail sont le reflet d'une expérimentation ad hoc (Bohn, 1987). De même, l'amélioration continue des processus, qui s'inscrit dans une logique incrémentale, engendre de l'apprentissage opérationnel. Ces trois variables forment donc un construit de l'apprentissage opérationnel.

La qualité de la représentation des variables sur les trois axes factoriels est bonne. Comme on peut le voir sur le tableau 3, la qualité globale de la représentation est de $67 \%$; le premier axe à lui seul expliquant environ $40 \%$ de la variance totale. Par ailleurs, toutes les variables, excepté la " modification des variables d'action », sont bien représentées : entre $58 \%$ et $85 \%$. Cette analyse nous donne des résultats très intéressants. Nous constatons en effet que les trois dimensions du processus d'apprentissage, mis en exergue dans l'étude de Mukherjee et al. (1998), s'y dégagent nettement. Notre première hypothèse, selon laquelle les construits de l'apprentissage organisationnel sont les mêmes dans les deux référentiels (ISO et TQM), est donc valide. 
Lorsque nous réalisons une analyse en composantes principales sur les processus de réalisation, au nombre de 84 , les trois dimensions qui caractérisent le processus d'apprentissage organisationnel s'y dégagent également.

\section{Impact des construits}

\section{de l'apprentissage sur la performance}

des processus : régression multiple sur les coordonnées factorielles

Dans cette section, nous allons déterminer l'impact des différents construits d'apprentissage sur la performance des processus ISO. La notion de performance et la nature de celle-ci font souvent l'objet de débats au sein de la communauté des chercheurs. Tout processus transforme des données d'entrée en données de sorite. La performance du processus peut donc se mesurer à travers ses outputs. Mais les indicateurs qui permettent d'appréhender la performance ne font pas l'unanimité. La norme ISO 9000 version 2000 demande des «mesures, analyses et améliorations » sans lister les indicateurs de mesure à retenir. Les indicateurs de performance dépendent donc de la nature des processus et de ce que le manager juge plus caractéristique de la performance du processus. Par exemple, la norme stipule que « l'organisme doit surveiller les informations relatives à la perception du client sur le niveau de satisfaction de ses exigences par l'organisme comme une des mesures de la performance du système de management de la qualité ». Mais si cette mesure paraît satisfaisante pour appréhender la performance du processus commercial, des indicateurs financiers sont par exemple nécessaires pour des processus liés au management des ressources. D'autres indicateurs peuvent être utilisés pour mesu- rer la performance des processus de production: productivité, nombre de rebuts, coût de production unitaire, etc. Kaplan et Norton (1998) proposent le tableau de bord prospectif permettant d'appréhender la performance de l'organisation sur quatre axes principaux : financier, client, processus internes, apprentissage organisationnel.

Nous n'avons donc pas cherché à définir la nature de la performance mais plutôt à estimer le niveau de performance du processus, étant sous-entendu ce que le manager entend par performance et les indicateurs qu'il utilise pour la mesurer.

La mesure ne porte pas non plus directement sur la performance du produit ou service ni sur celle de l'organisation dans son ensemble. Comme le note Shiba (1997), un des objectifs majeurs d'une organisation qui entreprend une démarche qualité est l'amélioration continue de ses produits et services. Pour y parvenir cependant, elle doit mettre l'accent sur les processus qui permettent d'obtenir ces produits et services. Cette approche permet de mettre en exergue la performance et l'efficacité de chaque processus, sa valeur ajoutée à l'organisation et de l'améliorer en permanence grâce à des mesures objectives; ce qui mènera naturellement à une performance d'ensemble de l'organisation.

Nous remarquons que les performances des processus, telles que les responsables qualité l'entendent, sont bonnes dans l'ensemble. Nous avons en effet des moyennes toujours supérieures à 0,6 pour les variables « atteinte des objectifs à court terme » et « atteinte des objectifs à long terme ». Il n'empêche que nous devons chercher à identifier les sources de telles performances. Cela nous permettra de leur accorder l'attention qu'elles méritent. Ci- 
dessous, nous montrons que la performance des processus est attribuable, en grande partie, à l'apprentissage organisationnel. L'étude de l'impact des construits de l'apprentissage sur la performance des processus, à travers la méthode de la régression multiple sur les coordonnées factorielles, nous donne en effet les résultats qui sont résumés dans le tableau 4 ci-dessous.

Impact des types d'apprentissage sur la performance à court terme des processus

Comme le montre le tableau 4, l'atteinte des objectifs à court terme dépend de l'apprentissage opérationnel. L'impact de l'apprentissage opérationnel sur l'atteinte des objectifs à court terme est non seulement important, mais également significatif à $1 \%$.

Atteinte des objectifs à court terme = $3,56+0,40 \mathrm{x}$ apprentissage opérationnel.
Le même résultat a été trouvé par Mukherjee et al. (1998). Quant à l'apprentissage conceptuel, il n'a pas un impact significatif sur l'atteinte des objectifs à court terme. Ce résultat montre clairement que l'apprentissage opérationnel est orienté exploitation. Rappelons que dans notre étude, l'axe de l'apprentissage opérationnel était fortement corrélé à la mise à jour des procédures, la mise à jour des instructions de travail et l'amélioration continue des processus. De telles pratiques, assez courantes lors des démarches qualité, sont certes indispensables, mais n'ont pas pour objectif d'ouvrir de nouvelles pistes de recherches technologiques, pouvant conduire à des découvertes tout à fait nouvelles (dépôt de brevets par exemple), susceptibles d'améliorer la performance à long terme des processus et de 1'organisation.

\section{Tableau 4}

IMPACT DES CONSTRUITS DE L'APPRENTISSAGE SUR LA PERFORMANCE DE 132 PROCESSUS

\begin{tabular}{|l|c|c|c|}
\hline & $\begin{array}{c}\text { Atteinte des objectifs } \\
\text { à court terme }\end{array}$ & $\begin{array}{c}\text { Atteinte des objectifs } \\
\text { à long terme }\end{array}$ & $\begin{array}{c}\text { Capacité à spécifier } \\
\text { l'impact }\end{array}$ \\
\hline Constante & $\begin{array}{c}3,56^{* *} \\
(0,11)\end{array}$ & $\begin{array}{c}3,81^{* *} \\
(0,14)\end{array}$ & $\begin{array}{c}3,62 * * \\
(0,13)\end{array}$ \\
\hline Facteur scope & 0,21 & 0,36 & 0,06 \\
$(0,14)$ & $(0,14)$ \\
\hline Apprentissage conceptuel & $(0,11)$ & $0,44^{* *}$ & $0,58^{* *}$ \\
$(0,14)$ & $(0,13)$ \\
\hline Apprentissage opérationnel & $(0,12)$ & $0,53 * *$ & 0,05 \\
$(0,14)$ & $0,13)$ \\
\hline $\mathrm{R}^{2}$ ajusté & $(0,11)$ & 0,35 & 0,27 \\
\hline Taille de l'échantillon & 0,20 & 132 & 132 \\
\hline
\end{tabular}

Note: $* *$ : significatif à $1 \%$; entre parenthèses : erreur standard. 


\section{Impact des types d'apprentissage sur la} performance à long terme des processus

$\mathrm{Si}$ on peut atteindre les objectifs à court terme en se focalisant sur l'apprentissage opérationnel, orienté exploitation des anciennes découvertes, l'atteinte des objectifs à long terme exige que l'organisation explore de nouvelles pistes de recherche, tout en étant capable de mettre en œuvre les découvertes déjà réalisées (voir tableau 4). Notre étude montre que l'apprentissage conceptuel joue un rôle certain sur la performance à long terme (niveau de signification < à $1 \%$ ). Mais à lui seul, il ne permet pas de réaliser les objectifs à long terme. Il permet certes de comprendre les vrais causes des problèmes et d'explorer de nouvelles pistes de recherche, pouvant conduire à la conception de nouveaux produits ou à une amélioration radicale des anciens produits. Mais une attitude extrême d'exploration technologique, en négligeant la mise en œuvre effective et l'exploitation des découvertes ainsi faites, place l'organisation dans une position de « rêveuse » (Miller, 1993): un dosage équilibré entre apprentissages conceptuel et opérationnel est indispensable pour la bonne réalisation des objectifs à long terme de l'organisation:

Atteinte des objectifs à long terme $=$ $3,81+0.44 \times$ apprentissage conceptuel $+0,53 \times$ apprentissage opérationnel.

En fait l'apprentissage conceptuel, à lui seul, permet surtout de comprendre l'impact des données d'entrée sur les résultats.

Rôle des construits de l'apprentissage sur la capacité à spécifier l'impact des inputs sur les outputs

Les résultats de la régression multiple (cf. tableau 4 ci-dessus) montrent clairement que la « capacité à spécifier l'impact » des inputs sur les outputs dépend de l'apprentissage conceptuel:

Capacité à spécifier l'impact $=$ $3.62+0.58 \times$ apprentissage conceptuel.

Ce résultat montre qu'il est possible de prévoir la variation de la qualité des produits suite à des changements dans les variables d'entrées (matières premières par exemple), grâce à l'apprentissage conceptuel. Cette meilleure maîtrise est importante dans la mesure où des variations dans la qualité des inputs n'entraîneront pas des dérives de production préjudiciables à l'entreprise. Par exemple, une des entreprises du secteur de l'électronique de puissance nous confiait n'être pas en mesure de prévoir l'effet des paramètres d'entrée de son processus de développement et de réalisation sur le produit final. Dans de telles situations, certaines modifications au niveau des variables d'entrée peuvent conduire à des dérives et résulter sur des produits ne respectant pas les limites de tolérance.

Contrairement aux résultats de Mukherjee et al. (1998), nous ne trouvons pas un impact significatif du champ de l'apprentissage (facteur scope) sur la capacité à spécifier l'impact des données d'entrée sur les résultats de processus. En fait, le facteur scope permet de diffuser les apprentissages de types conceptuel et opérationnel, créés localement, à toute l'organisation. Il facilite le passage de l'apprentissage individuel à l'apprentissage de groupe et organisationnel et peut donc avoir un effet indirect sur la performance. Comparativement à l'étude de Mukherjee et al. (1998), la qualité de la liaison entre les construits de l'apprentissage et nos variables de mesure de la performance est moindre. À l'exception des résultats sur l'atteinte des objectifs à long 
terme dont le coefficient de détermination $\left(\mathrm{R}^{2}\right)$ ajustée atteint 0,35 , la qualité des deux autres liaisons dépasse à peine 0,20.

Notre deuxième hypothèse stipule que l'impact des types d'apprentissage sur la performance des processus peut dépendre du type de processus pris en considération (processus de réalisation, de management ou support). Les analyses faites suivant la nature des processus montrent au contraire que l'impact des types d'apprentissage reste le même indépendamment de la nature des processus. Cette deuxième hypothèse n'est donc pas valide. Toutefois, des études prenant en compte un plus grand nombre de processus sont nécessaires pour confirmer ou non, sur des bases plus solides, cette hypothèse. En effet, à l'exception des processus de réalisation au nombre de 84 , les processus de management et les processus support n'étaient pas en nombre suffisant pour permettre d'obtenir des statistiques fiables.

\section{CONCLUSION}

Partant de l'hypothèse que les construits de l'apprentissage organisationnel sont les mêmes dans les entreprises certifiées ISO ou suivant des programmes TQM, et à l'aide de la méthode de l'analyse en composantes principales appliquée à 132 processus industriels issus d'entreprises alsaciennes, nous avons d'abord identifié trois dimensions ou construits qui caractérisent le processus d'apprentissage organisation- nel dans les entreprises certifiées ISO: un apprentissage opérationnel, un apprentissage conceptuel et un facteur de transfert et de diffusion des connaissances (scope). Nous avons ensuite déterminé l'impact de chaque construit sur la performance des processus, et ainsi montré que l'apprentissage opérationnel permet l'atteinte des objectifs à court terme et que la réalisation des objectifs à long terme requiert à la fois de l'apprentissage opérationnel et conceptuel. Cette étude confirme dans une large mesure celle menée aux États-Unis dans le cadre du TQM. La première implication managériale de notre étude est qu'elle invite les managers à développer aussi bien l'apprentissage opérationnel que conceptuel pour une meilleure performance de leurs organisations. Secundo, une fois créées, les connaissances ont besoin de structure et de cadre propice à leur transfert et à leur partage. Lorsqu'elle identifie des processus interfonctionnels, l'approche processus de l'organisation met l'accent sur les interrelations et favorise les synergies entre les processus. Elle constitue alors un moyen qui permet le transfert et le partage des connaissances, au même titre que les groupes de projets transfonctionnels, voire que les outils de knowledge management. De ce point de vue, la mise en place et le suivi des normes ISO 9000, au-delà de la maîtrise des processus, devraient faire l'objet d'une attention particulière du point de vue de la création de connaissance et de sa diffusion à travers l'organisation.

\section{Bibliographie}

Benezech D., Lambert G., Lanoux L., Lerch C., Loos-Baroin J., "Completion of knowledge Codification: an Illustration trough The ISO 9000 Standards Implementation Process", Research Policy, N-H Elsevier, n 30, 2001, p. 1395-1407. 
Boiral O., «ISO 9000, côté jardin et côté cour », Revue internationale de gestion, vol. 27, $\mathrm{n}^{\circ}$ 4, 2002, p. 34-42.

Bohn R. E., "Learning By Experimentation In Manufacturing”, Harvard Business school Working Paper 88-001, 1987.

Cole R. E., Managing Quality FADS: How American Business learned to play the quality game, Oxford University Press, Oxford, 1999.

Deming W. E., Hors de la crise, Edition Economica, Paris, 1991.

Drucker P. F., «L'émergence de la nouvelle organisation », Harvard Business Review, 1988 et Le knowledge management, Editions d'Organisations, Paris, 1999.

Dubreuil H., Le véritable intéressement des travailleurs à la vie de l'entreprise, Éditions de l'Entreprise Moderne, Paris, 1959.

Igalens J., «Perception par les DRH des liaisons entre quelques pratiques de gestion de la qualité et des indicateurs de mobilisation des ressources humaines », Revue Économie et Société, Série "Sciences de gestion », numéro spécial $\mathrm{XX}^{\mathrm{e}}$ anniversaire, août-septembre 1998, p. 169-180.

Ishikawa K., La gestion de la qualité: outils et applications pratiques, Dunod, Paris, 1984. ISO 9001 : 2000 (F), Systèmes de management de la qualité - Exigences.

Kim D. H., "The link between individual and organizational learning", Sloan Management Review, vol. 35, $\mathrm{n}^{\circ}$ 1, 1993, p. 37-50.

Lambert G., Loos-Baroin J., « L'impact de la certification ISO sur le management des connaissances: une étude de cas », Revue finance contrôle stratégie, mars 2004.

March J. G., "Exploration and exploitation in organizational learning", Organization science, vol. 2, $\mathrm{n}^{\circ} 1,1991$, p. 71-87.

Miller D., Le paradoxe d'Icare: comment les grandes entreprises se tuent à réussir, Eska, Paris, 1993.

Mukherjee A. S., Lapre M. A., Van Wassenhove L. N., "Knowledge driven quality improvement", Management Science, vol. 44, n 11, 1998.

Mukherjee A.S., Van Wassenhove L. N., "The impact of knowledge on quality", The practice of Quality Management, P. J. Lederer et U.S. Karmarkar eds, Kluwer Academic Publishers, Dordrecht, 1997.

Nonaka I., Takeuchi H., La connaissance créatrice, la dynamique de l'entreprise apprenante, De Boeck Université, Bruxelles, 1997.

Peters T., Thriving on Chaos: handbook for a management revolution, Pan, 1987.

Senge P. M., “The leader's new work: building learning organizations", Sloan Management Rev., Fall, 1990, p. 7-23.

Shewhart W. A., Les fondements de la maîtrise de la Qualité, Economica, Paris, 1989.

Shiba S., Graham A., Walden D., 4 révolutions du management par la qualité totale: manuel d'apprentissage et de mise en ceuvre du système TQM, Dunod, Paris, 1997.

Spencer B. A., "Models of organization and total quality management: a comparison and critical evaluation", Academy and Management Review, vol. 19, n 3, 1994, p. 446-471. 